\title{
Dark matter in the Kim-Nilles mechanism
}

\author{
Eung Jin Chun \\ Korea Institute for Advanced Study \\ Hoegiro 87, Dongdaemun-gu, \\ Seoul 130-722, Korea \\ Email)ejchun@kias.re.kr
}

\begin{abstract}
The Kim-Nilles mechanism relates the $\mu$ term with the axion scale $f_{a}$, leading to the axino-Higgsino-Higgs Yukawa coupling of order $\mu / f_{a}$. This can bring a dangerous thermal production of axinos. If the axino is stable, its mass has to be as small as $\mathcal{O}(0.1 \mathrm{keV})$, or the reheat temperature should be lower than $\mathcal{O}(10 \mathrm{GeV})$ taking the lower axion scale $10^{10} \mathrm{GeV}$ in order not to overclose the Universe. If the axino decays to a neutralino, the overproduced neutralinos can re-annihilate appropriately to saturate the observed dark matter density if the annihilation rate is of order $10^{-8} \mathrm{GeV}^{-2}$ for the axion scale larger than about $10^{11} \mathrm{GeV}$. Thus, a light Higgsinolike lightest supersymmetric particle with a sizable bino mixture becomes a good dark matter candidate whose nucleonic cross-section is larger than about $10^{-45} \mathrm{~cm}^{2}$.
\end{abstract}

\section{INTRODUCTION}

The strong CP problem is elegantly solved by the axion solution leading to the dynamical $\theta$ term:

$$
\mathcal{L}_{a}^{\mathrm{QCD}}=\frac{g_{s}^{2}}{32 \pi^{2}} \frac{a}{f_{a}} G_{\mu \nu}^{a} \tilde{G}_{a}^{\mu \nu},
$$

where $f_{a}$ is the axion decay constant allowed in the range of $10^{10} \mathrm{GeV} \lesssim f_{a} \lesssim 10^{12} \mathrm{GeV}[1]$. In the supersymmetric extension of the Standard Model, the origin of the supersymmetric Higgs mass term, $\mu$, can be related to the axion scale through the realization of the DFSZ axion [2]. This is the Kim-Nilles mechanism [3] which introduces the $\mu$ term interaction,

$$
W_{K N}=\lambda \frac{S^{2}}{M_{P}} H_{1} H_{2}
$$

where $M_{P}=2.4 \times 10^{18} \mathrm{GeV}$ is the reduced Planck mass. Recall that $S$ contains the axion degree of freedom and generates the axion scale through its vacuum expectation value at an intermediate scale, $\langle S\rangle \sim f_{a}$, and thereby induces the $\mu$ term: $\mu \sim f_{a}^{2} / M_{P}$. The superpartner of the axion, the axino $\tilde{a}$, gets massive upon supersymmetry breaking, and can be the lightest supersymmetric particle (LSP) to become a dark matter candidate. However, axinos can be efficiently produced from the thermal bath and typically their abundance is problematically large.

In the case of the KSVZ axion [4], the axino-gluino-gluon interaction, a supersymmetric counterpart of the axion-gluon-gluon interaction (1),

$$
\mathcal{L}_{\tilde{a}}^{Q C D}=\frac{g_{s}^{2}}{32 \pi^{2}} \frac{1}{f_{a}} \tilde{a} \sigma^{\mu \nu} \tilde{g}^{a} G_{\mu \nu}^{a}+\text { h.c. },
$$

is the main source for the thermal production of axinos either through freeze-out [5] or through regeneration [6 8]. Such processes are so efficient that the axino must be very 
light in order not to overclose the Universe unless the reheat temperature is below the electroweak scale to suppress its thermal production. Such a light axino requires special arrangements in the superpotential or supersymmetry breaking parameters to suppress tree and loop contributions to its mass which is generically of order the gravitino mass in gravity mediated supersymmetry breaking models $[9,10]$. On the other hand, smaller axino mass may arise more naturally in gauge mediation models [11, 12].

So far, the cosmic axino production has been studied extensively concentrating on the KSVZ axion model. In this paper, we consider the supersymmetric DFSZ axion model realized in the Kim-Nilles mechanism extending limited discussions in Refs. [13, 14]. The DFSZ axino production is led by the effective $\mu$ term interaction (2) which, after the field $S$ gets a vacuum expectation value, induces the axino-Higgsino-Higgs Yukawa term:

$$
\mathcal{L}_{\tilde{a}}^{K N}=c_{H} \frac{\mu}{f_{a}} \tilde{a}\left[\tilde{H}_{1} H_{2}+\tilde{H}_{2} H_{1}\right]+\text { h.c. },
$$

where $c_{H}$ is a model-dependent parameter of order one determined by the precise relation between $\langle S\rangle$ and $f_{a}$ and the axino fraction in the fermion component $\tilde{S}$.

As will be discussed in detail, the DFSZ axino interaction (4) is stronger than the QCD interaction (3) at low reheat temperature and thus the DFSZ axinos are also produced efficiently leading to a stringent mass bound or an strong upper limit on the reheat temperature below the electroweak scale when the axino is stable. On the other hand, if the DFSZ axino decays to a Higgsino and a Higgs boson, the decay occurs before thermal freeze-out of the usual neutralino LSP dark matter for lower values of $f_{a}$. For higher values of $f_{a}$, the DFSZ axino decays after the neutralino freeze-out and decay-produced neutralinos may overclose the Universe unless they re-annihilate sufficiently [15]. For a typical choice of the parameters, the neutralino annihilation rate of order $10^{-8} \mathrm{GeV}^{-2}$ turns out to lead to a right amount of dark matter abundance, which makes a light Higgsino LSP a natural dark matter candidate [16]. When the Higgsino-like dark matter contains a sizable fraction of bino, its spin-independent nucleonic scattering cross-section in the decoupling limit of heavy Higgs bosons and sfermions is shown to be of order $10^{-45} \mathrm{~cm}^{2}$ which is within the reach of future direct detection experiments.

\section{AXINO DARK MATTER}

A light DFSZ axino: Below the axion scale $f_{a}$, the axino-Higgsino-Higgs Yukawa interaction (44) is generated and plays a major role in producing the axino number density from thermal regeneration. The axino number density in unit of the entropy density, $Y_{\tilde{a}} \equiv$ $n_{\tilde{a}} / s$, is determined by solving the Boltzmann equation:

$$
z s H \frac{d Y_{\tilde{a}}}{d z}=\gamma,
$$

where $s=\left(2 \pi^{2} / 45\right) g_{*} T^{3}, H=0.33 \sqrt{g_{*}} T^{2} / M_{P}$ is the Hubble parameter, $g_{*}$ is the number of the degrees of freedom in thermal equilibrium, and $z \equiv m / T$ with the relevant mass parameter $m$ of the process $\gamma$. For our calculation of the axino production, we will consider decay (or inverse decay) processes coming from the $\mu$ term interaction (44) as their effect is expected to be larger than scattering processes when decay channels are open [17]. 
Let us first consider the decay process $H_{1} \rightarrow \tilde{a} \tilde{H}_{1}$ taking the Higgs mass $m_{H_{1}}$ larger than the Higgsino mass $\mu$ before the electroweak symmetry breaking. Integrating Eq. (5), one finds

$$
Y_{\tilde{a}} \approx \frac{135 g}{4 \pi^{4} g_{*}^{3 / 2}} \frac{\Gamma_{H_{1}} M_{P}}{m_{H_{1}}^{2}} \int_{0}^{\infty} z^{3} K_{1}(z) d z,
$$

where $g=4$ including the Higgs degrees of freedom and the final axino number, $\Gamma_{H_{1}} \approx$ $c_{H}^{2}\left(\mu / f_{a}\right)^{2} m_{H_{1}} / 8 \pi$ and $z=m_{H_{1}} / T$. This gives

$$
\Omega_{\tilde{a}}^{H} h^{2} \approx 0.11\left(\frac{m_{\tilde{a}}}{72 \mathrm{keV}}\right)\left(\frac{\mu}{500 \mathrm{GeV}}\right)\left(\frac{1 \mathrm{TeV}}{m_{H_{1}}}\right)\left(\frac{10^{11} \mathrm{GeV}}{f_{a} / c_{H}}\right)^{2},
$$

for $g_{*}=200$.

When the temperature of the Universe falls below the critical temperature, $T_{c} \sim 100$ $\mathrm{GeV}$, of the electroweak phase transition, new decay channels can open. In the decoupling limit of heavy Higgs bosons, the coupling of the lightest Higgs boson, $h$, resulting from the interaction (4) is

$$
\mathcal{L}_{\tilde{a}}^{h}=c_{H} \frac{\mu}{f_{a}} \tilde{a}\left[c_{\beta} \tilde{H}_{1}^{0}+s_{\beta} \tilde{H}_{2}^{0}\right] h+\text { h.c. },
$$

where the angle $\beta$ is defined by $t_{\beta}=\left\langle H_{2}^{0}\right\rangle /\left\langle H_{1}^{0}\right\rangle$. Assuming almost degenerate Higgsinos (with the mass $m_{\tilde{H}} \approx \mu$ ) decaying to $\tilde{a}+h$, one obtains the axino abundance:

$$
Y_{\tilde{a}} \approx \frac{135 g}{4 \pi^{4} g_{*}^{3 / 2}} \frac{\Gamma_{\tilde{H}} M_{P}}{m_{\tilde{H}}^{2}} \int_{z_{c}}^{\infty} z^{3} K_{1}(z) d z,
$$

where $z_{c}=m_{\tilde{H}} / T_{c}, g=2$ and $\Gamma_{\tilde{H}} \approx c_{H}^{2}\left(\mu / f_{a}\right)^{2} m_{\tilde{H}} / 16 \pi$. Taking $m_{\tilde{H}}=\mu=500 \mathrm{GeV}$ and $T_{c}=100 \mathrm{GeV}$ the integration in Eq. (9) from $z_{c}=5$ gives

$$
\Omega_{\tilde{a}}^{\tilde{H}} h^{2} \approx 0.11\left(\frac{m_{\tilde{a}}}{300 \mathrm{keV}}\right)\left(\frac{\mu}{m_{\tilde{H}}}\right)^{2}\left(\frac{10^{11} \mathrm{GeV}}{f_{a} / c_{H}}\right)^{2}
$$

for $g_{*}=100$. Another potentially important process for the DFSZ axino production comes from the supersymmetric top quark Yukawa coupling:

$$
\mathcal{L}_{\tilde{a}}^{t}=c_{t} \frac{m_{t}}{f_{a}}\left[\tilde{a} t \tilde{t}^{c}+\tilde{a} t^{\tilde{t}} \tilde{t}\right]+\text { h.c. },
$$

where $c_{t}$ quantifies the axino fraction in the Higgsino $\tilde{H}_{2}$. This opens up a new channel of the stop decay to an axino and the resulting axino population can be calculated as in Eq. (9) with a replacement of $\mu \rightarrow m_{t}, m_{\tilde{H}} \rightarrow m_{\tilde{t}, \tilde{t}^{c}}$ and $z_{c}=m_{\tilde{t}, \tilde{t}^{c}} / T_{c}$. One would get a similar result as in Eq. (10) except a more suppression of $\left(m_{t} / m_{\tilde{t}, \tilde{c}^{c}}\right)^{2}$ for $m_{\tilde{t}, \tilde{t}^{c}} \approx m_{\tilde{H}}$. We will use the stop decay for the case of a heavy axino.

The above results can be compared with the axino production from the QCD interaction (3) [8]:

$$
\Omega_{\tilde{a}}^{Q C D} h^{2} \approx 0.11\left(\frac{m_{\tilde{a}}}{44 \mathrm{MeV}}\right)\left(\frac{T_{R}}{10^{3} \mathrm{GeV}}\right)\left(\frac{10^{11} \mathrm{GeV}}{f_{a}}\right)^{2},
$$

where $T_{R}$ is the reheat temperature of the Universe. This shows that the KSVZ axino production is more effective than the DFSZ axino production for higher reheat temperature: 
$T_{R}>10^{5-6} \mathrm{GeV}$ for which the small loop factor $\left(g_{s}^{2} / 32 \pi^{2}\right)$ of the QCD interaction term (1) can be overcome. In any case, the axino mass must be much smaller than the electroweak scale which requires some special arrangement in the supersymmetric axion sector as mentioned in the Introduction.

A heavy DFSZ axino: When the axino mass takes its typical value of the soft supersymmetry breaking scale in gravity mediation, the above strong bounds can be evaded if the axino population is diluted by entropy dumping or the axino decays to a lighter dark matter candidate like the gravitino [14, 18]. The first possibility has been discussed in the context of thermal inflation [13]. In this case, the axino number density is suppressed by the Boltzmann factor and thus is quite sensitive to the reheat temperature after (thermal) inflation or any entropy dumping. To derive an appropriate reheat temperature for which the axino abundance saturates the required dark matter density of the Universe: $\Omega_{D M} h^{2} \approx 0.11$, let us take the axino production through the stop decay (11) after the electroweak symmetry breaking. Modifying the result (9), the axino number density is given by

$$
Y_{\tilde{a}} \approx \frac{135 g}{4 \pi^{4} g_{*}^{3 / 2}} \frac{\Gamma_{\tilde{t}} M_{P}}{m_{\tilde{t}}^{2}} \int_{z_{R}}^{\infty} z^{3} K_{1}(z) d z
$$

where $g=2, \Gamma_{\tilde{t}} \approx c_{t}^{2}\left(m_{t} / f_{a}\right)^{2} m_{\tilde{t}} / 8 \pi$ and $z_{R}=m_{\tilde{t}} / T_{R}$. Here the integration is essentially determined by the value of $z_{R}$ and the integration to the infinity is justified if the stop freeze-out temperature is smaller than $T_{R}$. For $z_{R} \gg 1$, the integration is well approximated by $\sqrt{\pi / 2} z_{R}^{5 / 2} \exp \left(-z_{R}\right)$. Thus, the axino relic density produced from the stop decay at low reheat temperature becomes

$$
\Omega_{\tilde{a}}^{\tilde{t}} h^{2} \approx 0.1\left(\frac{m_{\tilde{a}}}{500 \mathrm{GeV}}\right)\left(\frac{1 \mathrm{TeV}}{m_{\tilde{t}}}\right)\left(\frac{10^{11} \mathrm{GeV}}{f_{a}}\right)^{2}\left(\frac{z_{R}}{20.2}\right)^{5 / 2} e^{-z_{R}+20.2}
$$

for $m_{t}=172 \mathrm{GeV}, g_{*}=100$ and $c_{t}=1$. Taking $f_{a}=10^{10} \mathrm{GeV}$, one gets $z_{R}=25.4$ implying that the dangerous heavy axino relic density can be suppressed sufficiently for $T_{R} \lesssim 40 \mathrm{GeV}$. A stronger bound can be obtained, e.g., from the coupling (8) if a heavy Higgsino decay channel to the axino is open.

\section{LIGHT HIGGSINO DARK MATTER}

A more interesting possibility appears when a heavy axino is allowed to decay to the LSP without resorting to the above option of low reheat temperature. The DFSZ axino can decay to a usual neutralino through the $\mu$ term coupling (8) leading to overabundant population of the LSP dark matter. If the decay occurs before freeze-out of the dark matter, such a overproduction is harmless as the produced dark matter equilibrates and its relic density is determined by the usual freeze-out calculation [19]. Although the axino decays later, the overproduced dark matter population can get depleted appropriately through a strong re-annihilation and thus can settle down to the required value of $\Omega_{D M} h^{2} \approx 0.11$ [15].

In fact, the Kim-Nilles mechanism provides a natural framework to realize such a reannihilating dark matter scenario. As we will see, the annihilation rate of a light Higgsino LSP of order $10^{-8} \mathrm{GeV}^{-2}$ and the DFSZ axino decay rate turn out to be in the right range to make a good dark matter candidate the Higgsino LSP produced from the DFSZ axino decay $\tilde{a} \rightarrow h \tilde{H}$ for the axion scale of $f_{a} \approx 10^{11 \sim 12} \mathrm{GeV}$. 
Let us first consider if the heavy DFSZ axino population can be large enough. From the discussions in the previous section, the axino can be produced from the stop decay as in (13) but with the replacement of $z_{R} \rightarrow z_{c} \approx m_{\tilde{t}} / T_{c}$. This gives $Y_{\tilde{a}} \approx 5 \times 10^{-9}$ for $g_{*}=100$, $f_{a}=10^{11} \mathrm{GeV}, m_{\tilde{t}}=1 \mathrm{TeV}$ and $T_{c}=100 \mathrm{GeV}$. A more effective and inevitable process is the inverse decay $h \tilde{H} \rightarrow \tilde{a}$. From the couplings in Eq. (8), we find

$$
\begin{aligned}
Y_{\tilde{a}} & \approx \frac{135}{4 \pi^{4} g_{*}^{3 / 2}} \frac{\Gamma_{\tilde{a}} M_{P}}{m_{\tilde{a}}^{2}} \int_{z_{c}}^{\infty} z^{3} K_{1}(z) d z \\
& \sim 10^{-7}\left(\frac{\mu}{200 \mathrm{GeV}}\right)^{2}\left(\frac{10^{11} \mathrm{GeV}}{f_{a} / c_{H}}\right)^{2}\left(\frac{500 \mathrm{GeV}}{m_{\tilde{a}}}\right),
\end{aligned}
$$

for $g_{*}=100$ and $z_{c}=m_{\tilde{a}} / T_{c}=5$. This is more than $10^{4}$ times larger than the required value for a right dark matter density. If some decay mode is kinematically allowed before the electroweak symmetry breaking, such as $\tilde{a} \rightarrow H \tilde{H}$, the integration range of $z=[0, \infty]$ in Eq. (15) can be taken to increase the axino population by factor of 6 . Thus, we can safely conclude that the Higgsino dark matter population from the DFSZ axino decay is large enough to require re-annihilation.

Next question is whether the decay temperature of the axino is larger than the free-out temperature of the dark matter. The axino decay process $\tilde{a} \rightarrow h \tilde{H}$ ( $\tilde{H}$ may decay further to the LSP if it is not the LSP.) has the decay rate:

$$
\Gamma_{\tilde{a}} \approx \frac{c_{H}^{2}}{16 \pi}\left(\frac{\mu}{f_{a}}\right)^{2} m_{\tilde{a}}
$$

which corresponds to the decay temperature $T_{D}=g_{*}^{-1 / 4} \sqrt{3 \Gamma_{\tilde{a}} M_{P}}$. Defining $x_{D} \equiv m_{\chi} / T_{D}$ where $\chi$ denotes the LSP (the lighter Higgsino in our case), one gets

$$
x_{D} \approx 34\left(\frac{g_{*}}{70}\right)^{1 / 4}\left(\frac{500 \mathrm{GeV}}{m_{\tilde{a}}}\right)^{1 / 2}\left(\frac{m_{\chi}}{\mu}\right)\left(\frac{f_{a} / c_{H}}{10^{11} \mathrm{GeV}}\right) .
$$

Thus, requiring the decay temperature smaller than the freeze-out temperature $T_{f}$, that is, $x_{D}>x_{f}$ where $x_{f} \equiv m_{\chi} / T_{f} \approx 25$, one needs $f_{a} \gtrsim 7 \times 10^{10} \mathrm{GeV}$. Having $T_{D}<T_{f}$, the Boltzmann equation for the LSP abundance can be solved as [15]

$$
Y_{\chi}^{-1} \approx Y_{\chi}^{-1}\left(T_{D}\right)+\frac{\left\langle\sigma_{A} v\right\rangle s\left(T_{D}\right)}{H\left(T_{D}\right)}
$$

where the first term $Y_{\chi}\left(T_{D}\right)^{-1} \approx Y_{\tilde{a}}^{-1}$, as given in Eq. (15), is much smaller than the second term. Thus, the dark matter relic density is determined by

$$
\Omega_{\chi} h^{2}=\frac{10^{-11} \mathrm{GeV}^{-2}}{\left(g_{*} / 70\right)^{1 / 2}} \frac{x_{D}}{\left\langle\sigma_{A} v\right\rangle},
$$

which requires the dark matter annihilation rate: $\left\langle\sigma_{A} v\right\rangle=x_{D} \times 10^{-10} \mathrm{GeV}^{-2}$. From the relation (17), we get $x_{D} \approx 340$ for the (approximate) upper end of $f_{a} / c_{H}=10^{12} \mathrm{GeV}$ and the lower value of $x_{D}$ is put by $x_{f} \approx 25$. Therefore, we conclude that the annihilation rate in the range of

$$
2.5 \times 10^{-9} \mathrm{GeV}^{-2} \lesssim\left\langle\sigma_{A} v\right\rangle \lesssim 3.4 \times 10^{-8} \mathrm{GeV}^{-2}
$$


is required for the neutralino LSP. Such a large annihilation rate can be obtained indeed for the Higgsino-like LSP as can be seen from the general parameter scan made in Ref. [20] including co-annihilations. In our case, co-annihilation does not occur as the Higgsinos are not in thermal equilibrium and thus the heavier states decay away to the lighter ones.

For the calculation of the neutralino LSP annihilation rate in the mass range $200 \sim 500$ $\mathrm{GeV}$, we take the leading s-wave contributions in the decoupling limit of heavy Higgs bosons and all the sfermions. All the relevant terms can be found in Ref. [19] and let us recollect them in the below. We use the following convention for the diagonalization of the chargino and neutralino mass matrices:

$$
\begin{aligned}
\chi_{n}^{-} & =U_{n 1} \tilde{W}^{-}+U_{n 2} \tilde{H}_{1}^{-}, \\
\chi_{n}^{+} & =V_{n 1} \tilde{W}^{+}+V_{n 2} \tilde{H}_{2}^{+}, \\
\chi_{n}^{0} & =N_{11} \tilde{B}+N_{12} \tilde{W}^{3}+N_{13} \tilde{H}_{1}^{0}+N_{14} \tilde{H}_{2}^{0} .
\end{aligned}
$$

Note that we use the notation $\chi \equiv \chi_{1}^{0}$. The s-wave contributions to the annihilation rate in the $W^{ \pm} W^{\mp}, Z^{0} Z^{0}, Z^{0} h$ and $t \bar{t}$ final states are given by

$$
\begin{aligned}
\left\langle\sigma_{A} v\right\rangle_{W W} & =\pi \alpha_{2}^{2} \frac{\beta_{W W}^{3 / 2}}{m_{\chi}^{2}}\left[\sum_{n=1}^{2} \frac{\mathbf{U}_{n}^{2}+\mathbf{V}_{n}^{2}}{1+r_{\chi_{n}^{ \pm}}^{2}-r_{W}^{2}}\right]^{2}, \\
\left\langle\sigma_{A} v\right\rangle_{Z Z} & =\frac{\pi \alpha_{2}^{2}}{8 c_{W}^{4}} \frac{\beta_{Z Z}^{3 / 2}}{m_{\chi}^{2}}\left[\sum_{n=1}^{4} \frac{\mathbf{Z}_{n}^{2}}{1+r_{\chi_{n}^{0}}^{2}-r_{Z}^{2}}\right]^{2}, \\
\left\langle\sigma_{A} v\right\rangle_{Z h} & =\frac{\pi \alpha_{2}^{2}}{c_{W}^{2}} \frac{\beta_{Z h}^{3 / 2}}{m_{\chi}^{2}}\left[\sum_{n=1}^{4} \frac{-\mathbf{Z}_{n} \mathbf{T}_{n}\left(m_{\chi_{n}^{0}}-m_{\chi}\right)}{m_{Z}\left(1+r_{\chi_{n}^{0}}^{2}-r_{Z}^{2} / 2-r_{h}^{2} / 2\right)}+\frac{\mathbf{Z}_{0} m_{\chi}^{2}}{c_{W} m_{Z}^{2}}\right]^{2}, \\
\left\langle\sigma_{A} v\right\rangle_{t t} & =\frac{3 \pi \alpha_{2}^{2}}{16 c_{W}^{4}} \frac{\beta_{t t}^{1 / 2}}{m_{\chi}^{2}}\left[\mathbf{Z}_{0} \frac{m_{t} m_{\chi}}{m_{Z}^{2}}\right]^{2}
\end{aligned}
$$

where $r_{X} \equiv m_{X} / m_{\chi}$ and $\beta_{X Y} \equiv \sqrt{1-\left(r_{X}^{2}+r_{Y}^{2}\right) / 2-\left(r_{X}^{2}-r_{Y}^{2}\right) / 4}$. The couplings appearing in the above equations are defined as follows:

$$
\begin{aligned}
\mathbf{U}_{n} & =-\frac{1}{\sqrt{2}} N_{13} U_{n 2}+N_{12} U_{n 1}, \\
\mathbf{V}_{n} & =-\frac{1}{\sqrt{2}} N_{14} V_{n 2}+N_{12} V_{n 1}, \\
\mathbf{Z}_{n} & =-N_{13}^{2}+N_{14}^{2} \\
\mathbf{T}_{n} & =\frac{1}{2}\left(c_{\beta} N_{13}-s_{\beta} N_{14}\right)\left(N_{n 2}-t_{W} N_{n 1}\right)+(1 \leftrightarrow n) .
\end{aligned}
$$

The LSP annihilation rate is shown in Fig. 1 by blue solid contours in the $\mu-M_{1}$ plane assuming $M_{2}=2 M_{1}$ and $t_{\beta}=10$. Remark that the annihilation rates for the whole Higgsinolike LSP region (and the bino-like region with a large mixture of Higgsino) are in the range of $(1 \sim 3) \times 10^{-8} \mathrm{GeV}^{-2}$ which fits well into the desired range for re-annihilation (20). As can be expected, the most bino-like LSP region with a smaller Higgsino mixture $\left(\mu \gg M_{1}\right)$ is completely excluded as the annihilation rate becomes smaller than about $2 \times 10^{-9} \mathrm{GeV}^{-2}$ 


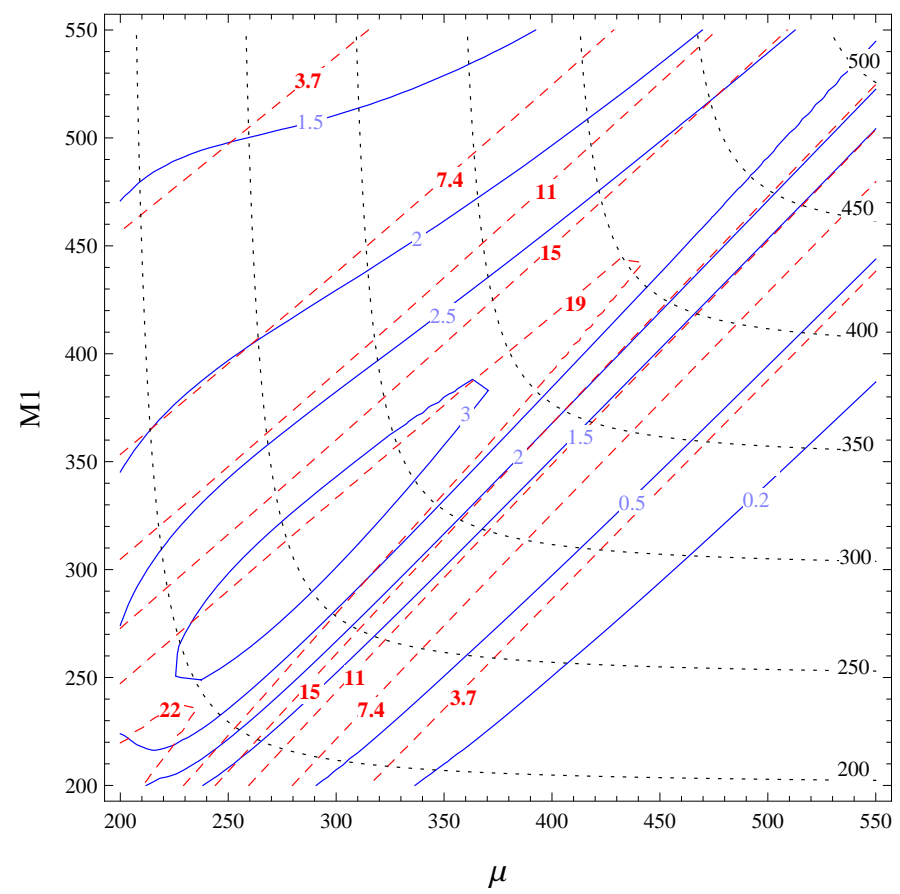

FIG. 1: In the $\mu-M_{1}$ plane showed is the LSP annihilation rate (blue solid contours) and the spinindependent nucleonic scattering cross-section (red dashed contours) in the unit of $10^{-8} \mathrm{GeV}^{-2}$ and $10^{-45} \mathrm{~cm}^{2}$, respectively. The dotted lines show the LSP mass. The calculation is made with the lightest Higgs mass $115 \mathrm{GeV}$, assuming $M_{2}=2 M_{1}$ and the decoupling limit of heavy Higgs bosons and all the sfermions. The nuclear form factor is taken to be $f_{T}=0.27$.

making $\Omega_{\chi} h^{2}>0.11$. In the same figure also shown is the spin-independent nucleonic cross-section which is calculated using the following formula [19]:

$$
\sigma_{S I}=4 \pi \alpha_{2}^{2} \frac{\mu_{n}^{2} m_{n}^{2}}{m_{W}^{2} m_{h}^{4}} \mathbf{T}_{1}^{2} f_{T}^{2}
$$

where $\mu_{n}$ is the reduced mass, $m_{n}$ is the nucleon mass, and $f_{T}$ is the nuclear form factor $f_{T} \equiv f_{T_{l q}}+2 f_{T_{G}} / 7$ with $f_{l q}=\sum_{q=u, d, s} f_{T_{q}}$ and $f_{T_{G}}=1-f_{T_{l q}}$. Note that the direct detection rate has a large uncertainty due to the nucleon form factor uncertainty (see, e.g., [21]). In our numerical calculation, we choose $f_{T}=0.27$ adopting the values of $f_{T_{u}}+f_{T_{d}}=0.05$, and $f_{T_{s}}=0.013$ from the recent lattice calculation [22].

The nucleon-LSP scattering depends sensitively on the bino fraction in $\mathbf{T}_{1}$ and the lightest Higgs boson mass. For the Higgs mass $m_{h}=115 \mathrm{GeV}$, the nucleonic scattering cross-section is shown to be as large as about $2 \times 10^{-44} \mathrm{~cm}^{2}$ for the Higgsino-like LSP with a large bino mixture in the mass range $m_{\chi}=(170 \sim 190) \mathrm{GeV}$.

Before closing this section, let us make remarks on the impact of the saxion $s$, the scalar partner of the axion [23], whose mass is expected to be order of the supersymmetry breaking scale $\sim 10^{2-3} \mathrm{GeV}$. In the DFSZ axion model [24], the dominant interaction terms of the saxion contain

$$
\mathcal{L}_{s}=\frac{s}{f_{a}}\left[\frac{\sqrt{2} x}{2} \partial_{\mu} a \partial^{\mu} a+c_{H} \mu\left(\tilde{H}_{1} \tilde{H}_{2}+\text { h.c. }\right)+c_{H}^{\prime} \mu^{2} \frac{h^{2}}{2}\right]
$$


where the Heavy Higgs bosons are decoupled. Here, $x \equiv \sum_{i} q_{i}^{3} v_{i}^{2} / f_{a}^{2}$ depends on the charges $q_{i}$ and the vacuum expectation values $v_{i}$ of the Peccei-Quinn symmetry breaking fields $S_{i}$ [10], and $c_{H}^{\prime}$ parameterizes the dependence on soft supersymmetry breaking terms. Note that the axion scale is given by $f_{a}=\sqrt{\sum_{i} q_{i}^{2} v_{i}^{2}}$. Like the axino, the saxion population can be produced thermally from the second and third terms in Eq. (25). When the decay of the saxion to the Higgsinos, $s \rightarrow \tilde{H} \tilde{H}$, is allowed, its inverse decay determines the thermal saxion population as

$$
\begin{aligned}
Y_{s} & \approx \frac{405}{8 \pi^{3} g_{*}^{3 / 2}} \frac{\Gamma_{s \rightarrow \tilde{H} \tilde{H}_{P}} M_{P}}{m_{s}^{2}} \\
& \approx 2.5 \times 10^{-6}\left(\frac{\mu}{200 \mathrm{GeV}}\right)^{2}\left(\frac{10^{11} \mathrm{GeV}}{f_{a} / c_{H}}\right)^{2}\left(\frac{500 \mathrm{GeV}}{m_{s}}\right),
\end{aligned}
$$

with $g_{*}=100$ and the decay rate $\Gamma_{s \rightarrow \tilde{H} \tilde{H}}=c_{H}^{2}\left(\mu / f_{a}\right)^{2} m_{s} / 4 \pi$. If the Higgsino channel is not open, the saxion decay to the Higgs bosons, $s \rightarrow h h$, from the third term in Eq. (25) becomes the main source for the thermal saxion production which leads to the saxion abundance comparable to Eq. (15). The saxion population can arise also from a coherent oscillation driven by an initial misalignment, $s_{i}$ :

$$
Y_{s} \approx 6.7 \times 10^{-7}\left(\frac{500 \mathrm{GeV}}{m_{s}}\right)^{1 / 2}\left(\frac{s_{i}}{10^{12} \mathrm{GeV}}\right)^{2}
$$

if the reheat temperature is high enough [25]. When the reheat temperature is low, $T_{R} \lesssim 10^{9}$ $\mathrm{GeV}$, the coherent production is typically smaller than the thermal production (26). The contribution of Eq. (27) can be larger than the thermal contribution (26) if $s_{i} \sim f_{a} \sim 10^{12}$ $\mathrm{GeV}$, and thus the LSP dark matter can mostly come from the saxion decay.

Contrary to the axino case, however, the overproduction problem of the LSP dark matter from the saxion decay is model-dependent. First of all, there is no such a problem when the saxion decay to the LSP is forbidden kinematically. Although it is allowed, the saxion may mainly decay to the axions through the first term in Eq. (25) giving the decay rate $\Gamma_{s \rightarrow a a}=x^{2} m_{s}^{3} / 64 \pi f_{a}^{2}$. In this case, the produced axions are red-shifted away and the LSP dark matter production is suppressed by the factor $\Gamma_{s \rightarrow \tilde{H} \tilde{H}} / \Gamma_{s \rightarrow a a} \approx 16 c_{H}^{2} \mu^{2} / x^{2} m_{s}^{2}$.

\section{CONCLUSION}

Dark matter property is discussed in the Kim-Nilles mechansim which realizes the DFSZ axion solution to the strong CP problem and solves the $\mu$ problem at the same time. The DFSZ axino regeneration by the $\mu$ term interactions (which is independent of the reheat temperature) is more efficient than the KSVZ axino regeneration from the QCD interaction at the reheat temperature of order $\mathrm{TeV}$, and thus puts also a stringent bound on the stable axino mass. The DFSZ axino dark matter mass can vary from $\mathcal{O}(0.1 \mathrm{keV})$ and $\mathcal{O}(10 \mathrm{MeV})$ depending on the allowed decay processes and the axion scale $f_{a} \approx 10^{10} \sim 10^{12} \mathrm{GeV}$. When the stable axino has the mass of order the electroweak scale, the reheat temperature has to be low enough to suppress the regeneration rate and, of course, dark matter can consist of heavy axinos if the reheat temperature is appropriately adjusted.

The DFSZ axino may decay to a neutralino through the $\mu$ term and the overproduced neutralinos can be thermalized or re-annihilate sufficiently to saturate the observed dark 
matter density. A light Higgsino-like LSP is shown to have the annihilation rate $(1 \sim$ $3) \times 10^{-8} \mathrm{GeV}^{-2}$ and thus becomes a good dark matter candidate realizing the re-annihilation scenario for the axion scale $f_{a} \approx 10^{11 \sim 12} \mathrm{GeV}$. Furthermore, the Higgsino-like LSP with a sizable mixture of the bino component has the spin-independent nucleonic cross-section as large as $2 \times 10^{-44} \mathrm{~cm}^{2}$ for the Higgs mass $115 \mathrm{GeV}$, and thus might be observed in the future direct detection experiments.

Acknowledgments: This work was supported by Korea Neutrino Research Center through National Research Foundation of Korea Grant (2009-0083526). The author thanks Ki-Young Choi for discussions and careful reading of the manuscript.

[1] For a recent review, see, J. E. Kim and G. Carosi, Rev. Mod. Phys. 82, 557 (2010) arXiv:0807.3125 [hep-ph]].

[2] M. Dine, W. Fischler and M. Srednicki, Phys. Lett. B 104, 199 (1981); A. R. Zhitnitsky, Sov. J. Nucl. Phys. 31, 260 (1980) [Yad. Fiz. 31, 497 (1980)].

[3] J. E. Kim and H. P. Nilles, Phys. Lett. B 138, 150 (1984).

[4] J. E. Kim, Phys. Rev. Lett. 43, 103 (1979); M. A. Shifman, A. I. Vainshtein and V. I. Zakharov, Nucl. Phys. B 166, 493 (1980).

[5] K. Rajagopal, M. S. Turner and F. Wilczek, Nucl. Phys. B 358, 447 (1991).

[6] L. Covi, H. B. Kim, J. E. Kim and L. Roszkowski, JHEP 0105, 033 (2001) arXiv:hep-ph/0101009.

[7] A. Brandenburg and F. D. Steffen, JCAP 0408, 008 (2004) arXiv:hep-ph/0405158.

[8] A. Strumia, JHEP 1006, 036 (2010) arXiv:1003.5847 [hep-ph]].

[9] T. Goto and M. Yamaguchi, Phys. Lett. B 276, 103 (1992); E. J. Chun, J. E. Kim and H. P. Nilles, Phys. Lett. B 287, 123 (1992) arXiv:hep-ph/9205229].

[10] E. J. Chun and A. Lukas, Phys. Lett. B 357, 43 (1995) arXiv:hep-ph/9503233.

[11] E. J. Chun, Phys. Lett. B 454, 304 (1999) arXiv:hep-ph/9901220]; E. J. Chun and H. B. Kim, Phys. Rev. D 60, 095006 (1999) arXiv:hep-ph/9906392.

[12] K. S. Jeong and M. Yamaguchi, arXiv:1102.3301 [hep-ph].

[13] E. J. Chun, H. B. Kim and D. H. Lyth, Phys. Rev. D 62, 125001 (2000) arXiv:hep-ph/0008139.

[14] K. Choi, E. J. Chun, H. D. Kim, W. I. Park and C. S. Shin, arXiv:1102.2900 [hep-ph].

[15] K. Y. Choi, J. E. Kim, H. M. Lee and O. Seto, Phys. Rev. D 77, 123501 (2008) arXiv:0801.0491 [hep-ph]].

[16] H. Baer, A. Lessa, S. Rajagopalan and W. Sreethawong, arXiv:1103.5413 [hep-ph].

[17] For some details of the calculation, see, L. J. Hall, K. Jedamzik, J. March-Russell and S. M. West, JHEP 1003, 080 (2010) [arXiv:0911.1120 [hep-ph]].

[18] C. Cheung, G. Elor and L. J. Hall, arXiv:1104.0692 [hep-ph].

[19] For a review, see, G. Jungman, M. Kamionkowski and K. Griest, Phys. Rept. 267, 195 (1996) arXiv:hep-ph/9506380].

[20] J. Edsjo and P. Gondolo, Phys. Rev. D 56, 1879 (1997) arXiv:hep-ph/9704361.

[21] A. Bottino, F. Donato, N. Fornengo and S. Scopel, Astropart. Phys. 13, 215 (2000) arXiv:hep-ph/9909228.

[22] K. Takeda, S. Aoki, S. Hashimoto, T. Kaneko, T. Onogi and N. Yamada [JLQCD Collaboration], PoS LATTICE2010, 160 (2010) [arXiv:1012.1907 [hep-lat]]. 
[23] J. E. Kim, Phys. Rev. Lett. 67, 3465 (1991).

[24] For a recent discussion on the saxion in the KSVZ model, see, H. Baer, S. Kraml, A. Lessa and S. Sekmen, JCAP 1104, 039 (2011) arXiv:1012.3769 [hep-ph]].

[25] S. Chang and H. B. Kim, Phys. Rev. Lett. 77, 591 (1996) arXiv:hep-ph/9604222; M. Kawasaki, K. Nakayama and M. Senami, JCAP 0803, 009 (2008) arXiv:0711.3083 [hep$\mathrm{ph}]$. 